NASZA DERMATOLOGIA Online OUR DERMATOLOGY Online Nil

Competing Interests: None

\section{A STUDY ON EFFICACY OF ORAL ZINC THERAPY FOR TREATMENT OF ACRODERMATITIS ENTEROPATHICA}

\author{
Neerja Puri \\ Department of Dermatology and Venereology, Punjab Health Systems Corporation, \\ Ferozepur, Punjab, India
}

Corresponding author: Dr. Neerja Puri neerjaashu@,rediffmail.com

\begin{abstract}
Introduction: Acrodermatitis enteropathica (AE) is a disorder of zinc metabolism that occurs in one of two forms: an inborn (congenital) form and an acquired form. The inborn form of $\mathrm{AE}$ is a rare genetic disorder characterized by intestinal abnormalities that leads to inability to absorb zinc from the intestine. The lack of zinc presents, characteristically, as skin inflammation with pustules occurring around the mouth and/or anus, diarrhea.

Aims: To study the efficacy of oral zinc therapy on thirty patients of acrodermatitis enteropathica.

Methods: Thirty clinically diagnosed patients of acrodermatitis enteropathica were taken for the study. The criterion of diagnosis of this condition was the clinical picture of symmetrical vesiculo-pustular dermatitis, in upper and lower limbs and periorificial regions.

Results and Discussion: In our study maximum (50\%) patients were below 1 year of age, 33.3\% patients were between $13-24$ months of age, $6.66 \%$ patients were between 25- 36 months and 37- 48 months each and 3.33\% patients were between $49-60$ months of age. Nail changes were seen in $60 \%$ children. Also, it was seen that perioral area was the commonest (86.6\%) site involved, followed by anogenital area in $80 \%$ patients, palms and soles were involved in $66.6 \%$ patients, arms in $46.6 \%$ patients and legs were seen in $40 \%$ patients. Regarding clinical features, dermatitis was seen in $100 \%$ patients, alopecia was seen in $40 \%$ patients, diarrhea in $60 \%$ patients and mental disturbances were seen in $30 \%$ patients.
\end{abstract}

Key words: acrodermatitis; enteropathica; zinc

\section{Introduction}

Acrodermatitis enteropathica is a rare genetic disorder characterised by diarrhoea, an inflammatory rash around the mouth and/or anus and hair los [1]. Acrodermatitis enteropathica is due to malabsorption of zinc through the intestinal cells. The precise cause is not known, but it may relate to mutations in a gene (SLC39A4) that codes the zinc transporter protein, ZIP4 [2,3]. It is thought that the missing protein may be responsible for decreased zinc uptake and abnormal zinc metabolism. To have congenital acrodermatitis enteropathica you must inherit two defective genes (one from each parent) i.e. the inheritance is autosomal recessive [4]. If an individual receives one normal gene and one defective gene, the person will be a carrier for the disease, but usually will not show symptoms. Symptoms usually occur in bottlefed infants within a few days or weeks after birth and breastfed infants soon after weaning. Both males and females are equally affected.

Zinc is an essential component of the diet. Zinc in human milk is more absorbable than that from infant formulas or cow's milk, hence the later onset of acrodermatitis enteropathica in breast-fed babies compared to formula-fed babies [5]. Zinc is also found in meat, shellfish and wheat germ. Foods of plant origin are mostly low in zinc. Phytates present in cereals and soy and high levels of calcium, can reduce the absorption of zinc through the duodenum. Zinc is needed to assist metalloenzymes that are involved in many cellular processes throughout the body [6,7]. These include the production of anti-inflammatory agents (cytokines and antioxidants) and the normal functioning of the brain. If zinc deficiency is suspected, the following investigations may be helpful [8].

- Serum/plasma zinc levels confirm the diagnosis (normal levels are 60-140 microgram/dL);

Urinary zinc excretion may be reduced;

Blood count may reveal anaemia;

Skin biopsy may show characteristic features.

Acrodermatitis enteropathica is characterized by chronic diarrhea which may be mild or severe, and the presence of fatty substances in the feces (steatorrhea) [9]. In the congenital form symptoms start gradually, frequently at the time of weaning of an infant. 
The skin around body openings such as the mouth, anus, and eyes; and the skin on elbows, knees, hands, and feet becomes inflamed. Skin lesions are usually blistered (vesicobullous) and after drying out become psoriasis-like. The skin around the nails may also be inflamed and the nail may be abnormal due to malnourished tissue [10]. Hair loss on the scalp, eyelids, and eyebrows may be total (alopecia). Inflammation of the membrane that lines the eyelid (conjunctivitis), usually also occurs.

\section{Material and Methods}

Thirty clinically diagnosed patients of acrodermatitis enteropathica were taken for the study. The criterion of diagnosis of this condition was the clinical picture of symmetrical vesiculo-pustular dermatitis, in upper and lower limbs and periorificial regions. Another diagnostic criteria was prompt response to oral zinc therapy.The patents selected were below 5 years of age. Written informed consent was taken from the parents of the children before the start of the study. Prior approval of the hospital ethical committee was taken for the study. The routine haemogram was performed of all the patients. Histopathological examination was done in those patients wherever diagnosis was in doubt. Serum zinc levels were calculated before the treatment was started. The children were put on oral zinc therapy, if serum zinc levels were found to be below $50 \mu \mathrm{gm} / \mathrm{dl}$. The children with zinc deficiency were given oral zinc supplements in the form of syrup zinc sulphate given in the dose of $5 \mathrm{mg} /$ $\mathrm{kg}$ body weight. The oral zinc was continued for 4weeks or till the clearance of dermatitis, whichever was earlier. Post treatment serum zinc levels were again estimated.

The specimens for plasma zinc were collected in plastic syringes or acid washed vacutainer tubes with no rubber stopper to prevent exogenous contamination that could lead to spuriously normal measurements. Plasma zinc concentrations of less than $50 \mu \mathrm{gm} / \mathrm{dl}$ were suggestive, but not diagnostic of acrodermatitis enteropathica.

\section{Results and Discussion}

The results were tabulated and the data was analysed. Maximum (50\%) patients were below 1 year of age, 33.3\% patients were between 13-24 months of age (Tabl. I).

Table II shows that there were $60 \%$ male children and $40 \%$ female children.

Nail changes were seen in $60 \%$ children. $60 \%$ patients showed nail ridging, $40 \%$ patients had paronychia and $6.66 \%$ patients had nail dystrophy (Tabl. III).

Table IV shows that perioral area was the commonest $(86.6 \%)$ site involved, followed by anogenital area in $80 \%$ patients, palms and soles were involved in $66.6 \%$ patients, arms in $46.6 \%$ patients and legs were seen in $40 \%$ patients. Cermatitis was seen in $100 \%$ patients, alopecia was seen in $40 \%$ patients, diarrhea in $60 \%$ patients and mental disturbances were seen in $30 \%$ patients (Tabl. V).

\begin{tabular}{|l|l|l|l|}
\hline \multicolumn{1}{|c|}{ Sr no } & \multicolumn{1}{|c|}{ Age (months) } & \multicolumn{1}{c|}{ Number } & \multicolumn{1}{c|}{ Percentage } \\
\hline 1 & $<12$ months & 15 & $50 \%$ \\
\hline 2 & $13-24$ & 10 & $33.3 \%$ \\
\hline 3 & $25-36$ & 2 & $6.66 \%$ \\
\hline 4 & $37-48$ & 2 & $6.66 \%$ \\
\hline 5 & $49-60$ & 1 & $3.33 \%$ \\
\hline & Total & 30 & 100 \\
\hline
\end{tabular}

\begin{tabular}{|l|l|l|l|}
\hline \multicolumn{1}{|c|}{ Sr No } & \multicolumn{1}{|c|}{ Sex } & \multicolumn{1}{c|}{ Number } & \multicolumn{1}{c|}{ Percentage } \\
\hline 1 & Male children & 18 & $60 \%$ \\
\hline 2 & Female children & 12 & $40 \%$ \\
\hline & Total & 30 & 100 \\
\hline
\end{tabular}

Table II. Sex distribution of children with acrodermatitis enteropathica

\begin{tabular}{|l|l|l|l|}
\hline \multicolumn{1}{|c|}{ Sr No } & \multicolumn{1}{|c|}{ Sex } & \multicolumn{1}{c|}{ Number } & \multicolumn{1}{c|}{ Percentage } \\
\hline 1 & Nail ridging & 18 & $60 \%$ \\
\hline 2 & Paronychia & 12 & $40 \%$ \\
\hline 3 & Nail dystrophy & 2 & $6.66 \%$ \\
\hline
\end{tabular}

Table III. Nail changes in patients with acrodermatitis enteropathica 


\begin{tabular}{|l|l|l|l|}
\hline \multicolumn{1}{|c|}{ Sr No } & \multicolumn{1}{|c|}{ Site } & \multicolumn{1}{c|}{ Number } & \multicolumn{1}{c|}{ Percentage } \\
\hline 1 & Perioral & 26 & $86.6 \%$ \\
\hline 2 & Anogenital & 24 & $80 \%$ \\
\hline 3 & Palms and soles & 20 & $66.6 \%$ \\
\hline 4 & Arms & 14 & $46.6 \%$ \\
\hline 5 & Legs & 12 & $40 \%$ \\
\hline 6 & Trunk & 8 & $26.6 \%$ \\
\hline
\end{tabular}

Table IV. Site of involvement of skin lesions

\begin{tabular}{|l|l|l|l|}
\hline \multicolumn{1}{|c|}{ Sr No } & Clinical Features & \multicolumn{1}{c|}{ Number } & \multicolumn{1}{c|}{ Percentage } \\
\hline 1 & Dermatitis & 30 & 100 \\
\hline 2 & Alopecia & 12 & 40 \\
\hline 3 & Diarrhoea & 18 & 60 \\
\hline 4 & Mental disturbances & 9 & 30 \\
\hline
\end{tabular}

Table V. Clinical features of acrodermatitis enteropathica

Acrodermatitis enteropathica is a rare inherited form of zinc deficiency, characterized by periorificial and acral dermatitis, alopecia, and diarrhea [11]. The inherited form of acrodermatitis enteropathica was usually fatal until the role of zinc was discovered in 1973. It should be treated with $1 \mathrm{mg} / \mathrm{kg}$ body wait of oral zinc supplementation per day for life. Zinc gluconate is better tolerated than zinc sulfate. Zinc can be given during pregnancy. After zinc replacement the skin lesions heal within one to two weeks, diarrhoea ceases and irritability and depression of mood improve within $24 \mathrm{hrs}$.Secondary bacterial and/or fungal infection of lesions require appropriate antibiotic therapy. Additionally, zinc deficiency can present in full-term breastfed infants as a result of low maternal serum zinc levels or a defect in mammary zinc secretion [12]. Thus, not all infants who have an acrodermatitis enteropathica-like presentation have the genetic disorder.The clinical findings of infant with acrodermatitis enteropathica are as follows: Infants are typically irritable and often inconsolable, and they show a slowing or cessation of growth and development.

The skin shows erythematous, dry and scaly patches and plaques which may evolve into crusted, vesiculobullous, erosive, psoriasiform, and pustular lesions. Lesions are predominantly distributed in a periorificial and acral pattern and may become secondarily infected with Staphylococcus aureus or Candida albicans. Red and inflamed patches of dry and scaly skin, particularly around body openings such as the mouth, anus, and eyes, and the skin on elbows, knees, hands, and feet. It may look like atopic dermatitis. Patches evolve into crusted, blistered, pus-filled and eroded lesions. There is usually a sharp demarcation between the affected area and normal skin. The mucosa shows angular cheilitis, glossitis, conjunctivitis, blepharitis, punctate keratopathy and photophobia. The nails shows paronychia and nai dystrophy is typical.Also the patients have loss of scalp hair, eyebrows, and eyelashes.

Other features of acrodermatitis enteropathica includes conjunctivitis, sensitivity to light, loss of appetite, diarrhoea, mild or severe, irritability (babies cry and whine incessantly), depressed mood and growth failure. Histological evaluation of a skin biopsy specimen is characteristic, but the same findings can be seen in other nutritional disorders [13]. The histological findings vary with the age of the lesion. Early lesions show confluent parakeratosis associated with a reduced granular layer. Often, exocytosis of neutrophils into the epidermis is noted, which may be acanthotic and exhibit slight spongiosis. The intracellular edema eventuates into pallor of the upper third of the epidermis. Subsequently, subcorneal and intraepidermal clefts may develop as a result of massive ballooning and reticular degeneration, with necrosis of the keratinocytes. In late lesions, psoriasiform hyperplasia of the epidermis and less epidermal pallor are noted.

In our study maximum (50\%) patients were below 1 year of age, $33.3 \%$ patients were between 13-24 months of age, $6.66 \%$ patients were between 25-36 months and 37-48 months each and $3.33 \%$ patients were between $49-60$ months of age. There were $60 \%$ male children and $40 \%$ female children. Nail changes were seen in $60 \%$ children. $60 \%$ patients showed nail ridging, $40 \%$ patients had paronychia and $6.66 \%$ patients had nail (Fig. 1, 2). These children had low body weight, were apathetic and irritable. Alopecia of scalp and eyebrow hair was seen in $38 \%$ children. Also, it was seen that perioral area was the commonest $(86.6 \%)$ site involved, followed by anogenital area (Fig. 3) in $80 \%$ patients, palms and soles were involved in $66.6 \%$ patients, arms in $46.6 \%$ patients and legs were seen in $40 \%$ patients. Regarding clinical features, dermatitis was seen in $100 \%$ patients, alopecia was seen in $40 \%$ patients, diarrhea in $60 \%$ patients and mental disturbances were seen in 30 $\%$ patients. Most of the children had low body weight,were apathetic and irritable. Out of 30 cases, one patient had intractable diarrhea and was severely dehydrated and died of electrolyte imbalance. Three cases had positive family history.Two of their siblings haad suffered from similar disease. Some of the children had anaemia and ascariasis infestation. In our study,clinical improvement started within 2 - 3 weeks of zinc supplementation (Fig. 4, 5). 


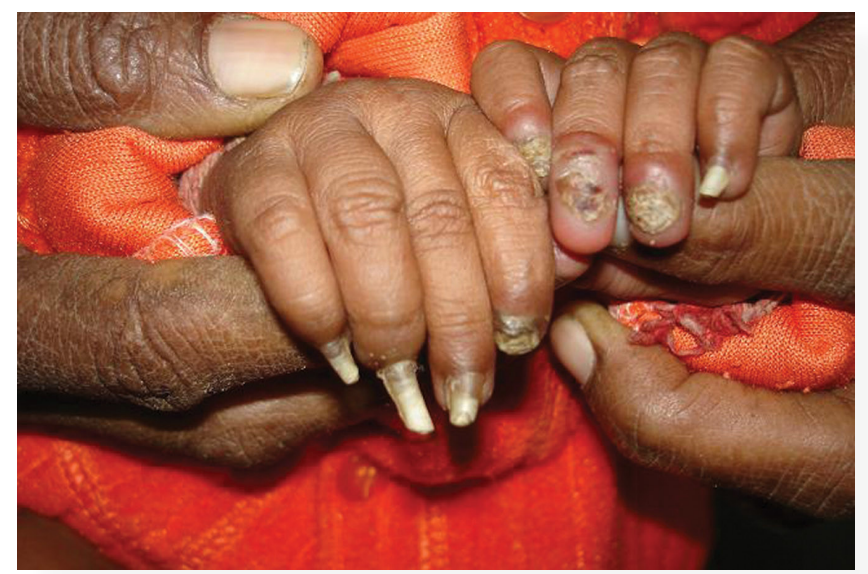

Figure 1. Finger nail dystrophy in a 3 months old child

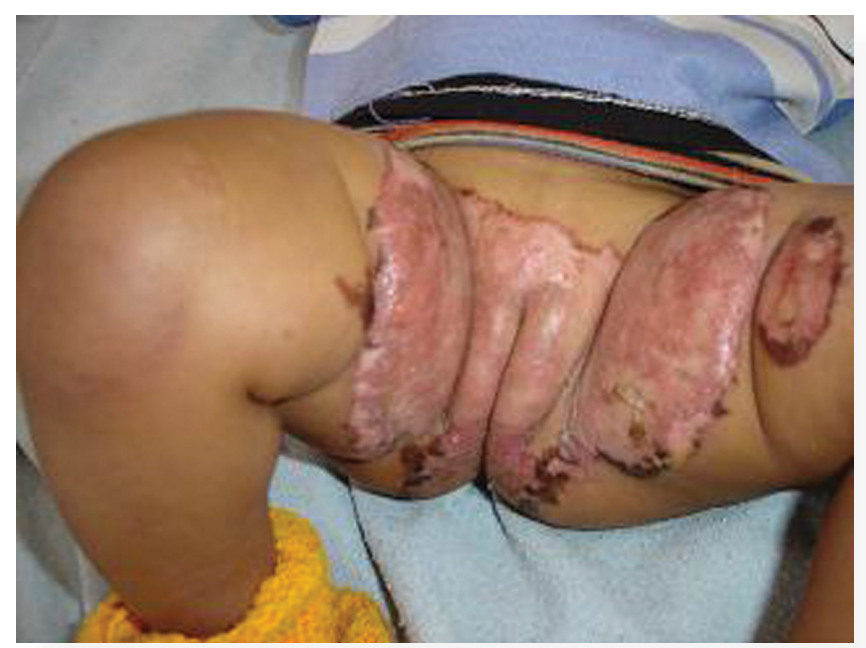

Figure 3. Erythematous plaque in a 7 months old female child

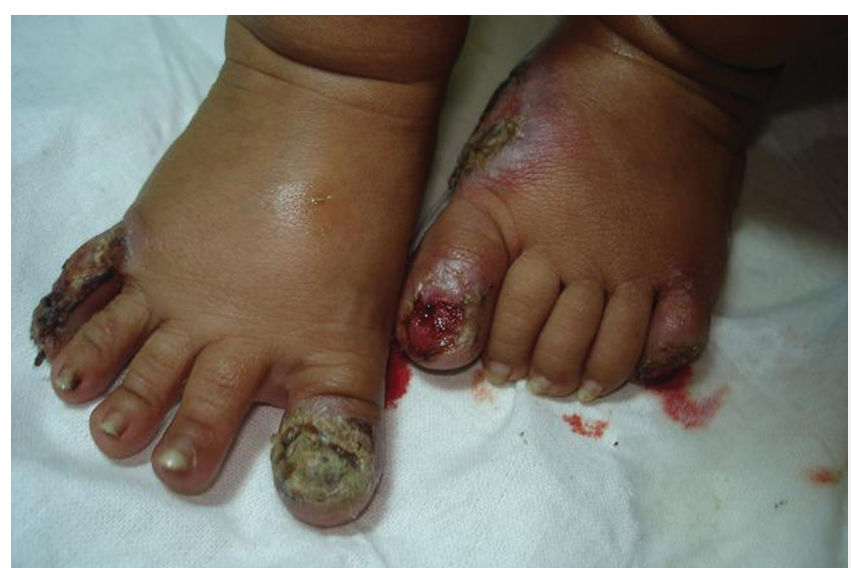

Figure 2. Toe nail with subungual haemorrhage in toe nails

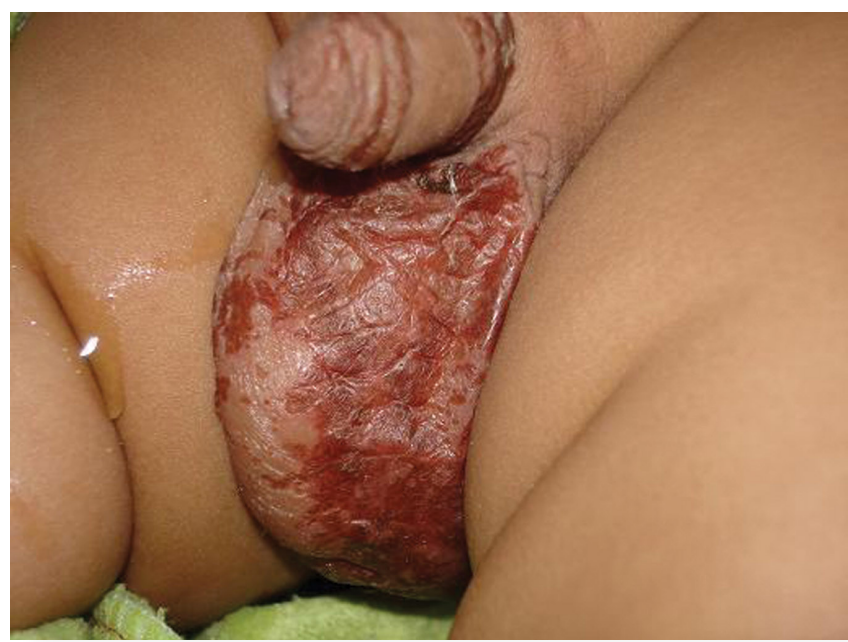

Figure 4. Erythema over the genital area in a 5 months old infant befeore treatment

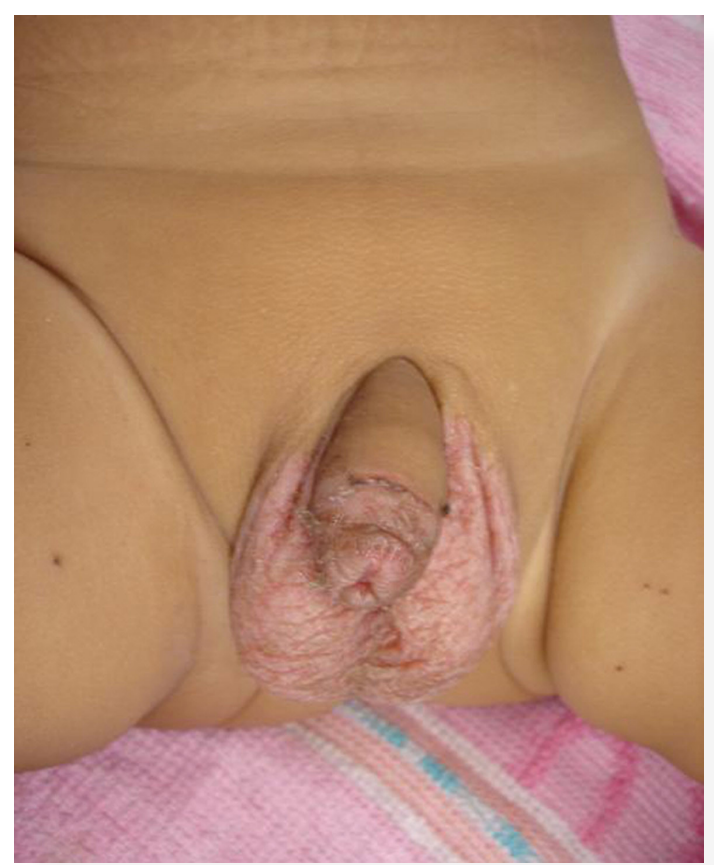

Figure 5. After treatment with zinc therapy in the same child 
Treatment of acrodermatitis enteropathica requires lifelong zinc supplementation. Acrodermatitis enteropathica is treated with zinc supplements in the form of zinc sulfate. These supplements should be given as soon as diagnosis of the disorder is made and they have to be continued for life. The drug Diodoquin (iodoquinol) is another treatment that usually clears up symptoms within a week. If the disorder is caused by intravenous feeding, adding zinc supplements to the nutritional regimen can prevent and/ or clear up manifestations of AE.Typically, 3-5 mg/ $\mathrm{kg}$ of zinc gluconate or sulfate is administered orally each day. Clinical improvement occurs prior to any significant change in the plasma zinc levels, usually within days to weeks of initiating treatment [14]. Monitor serum zinc levels and alkaline phosphatase values every 3-6 months $[15,16]$. Acrodermatitis enteropathica exacerbation during pregnancy or the stress of disease may require an increase in therapy. Warm compresses to remove the scale crust, followed by application of white petrolatum to eroded skin lesions, may enhance reepithelialization when used concurrently with zinc replacement [17]. Genetic counseling is recommended for families of patients with the congenital form of acrodermatitis enteropathica. Although no special diet is required for acrodermatitis enteropathica patients, as long as zinc supplementation is continued, certain foods contain increased levels of zinc, including oysters, crab, beef, pork, and fowl. Zinc content is directly related to protein $[18,19]$.

\section{REFERENCES}

1. Perafan-Riveros C, Franca LF, Alves AC, Sanches JA Jr: Acrodermatitis enteropathica: case report and review of the literature. Pediatr Dermatol. 2002;19:426-31.

2. Kury S, Dreno B, Bezieau S: Identification of SLC39A4, a gene involved in acrodermatitis enteropathica. Nat Genet. 2002;31:23940 .

3. Nakano A, Nakano H, Nomura K, Toyomaki Y, Hanada K: Novel SLC39A4 mutations in acrodermatitis enteropathica. J Invest Dermatol. 2003;120:963-6.
4. Wang K, Pugh EW, Griffen S, Doheny KF, Mostafa WZ, AlAboosi MM: Homozygosity mapping places the acrodermatitis enteropathica gene on chromosomal region 8q24.3. Am J Hum Genet. 2001;68:1055-60.

5. Maverakis E, Fung MA, Lynch PJ, Draznin M, Michael DJ, Ruben B: Acrodermatitis enteropathica and an overview of zinc metabolism. J Am Acad Dermatol. 2007;56:116-24.

6. Wang K, Zhou B, Kuo YM, Zemansky J, Gitschier J: A novel member of a zinc transporter family is defective in acrodermatitis enteropathica. Am J Hum Genet. 2002;71:66-73.

7. Evans GW, Johnson PE: Zinc-binding factor in acrodermatitis enteropathica. Lancet. 1976;2:131-3.

8. Connors TJ, Czarnecki DB, Haskett MI: Acquired zinc deficiency in a breast-fed premature infant. Arch Dermatol. 1983;119:319-21. 9. Perafán-Riveros C, França LF, Alves AC, Sanches JA Jr: Acrodermatitis enteropathica: case report and review of the literature. Pediatr Dermatol. 2002;19:426-31.

10. Sehgal VN, Jain S: Acrodermatitis enteropathica. Clin Dermatol. 2000;18:745-8.

11. Radja N, Charles-Holmes R: Acrodermatitis enteropathicalifelong follow-up and zinc monitoring. Clin Exp Dermatol. 2002;27:62-3.

12. Kiechl-Kohlendorfer U, Fink FM, Steichen-Gersdorf E: Transient symptomatic zinc deficiency in a breast-fed preterm infant. Pediatr Dermatol. 2007;24:536-40.

13. Gonzalez JR, Botet MV, Sanchez JL: The histopathology of acrodermatitis enteropathica. Am J Dermatopathol. 1982;4:303-11. 14. Sandstead HH: Understanding zinc: recent observations and interpretations. J Lab Clin Med. 1994;124:322-7.

15. Van Wouwe JP: Clinical and laboratory diagnosis of acrodermatitis enteropathica. Eur J Pediatr. 1989;149:2-8.

16. Prasad AS: Zinc: an overview. Nutrition. 1995;11:93-9.

17. Lombeck T, Schnippering HG, Ritzl F, Feinendegen LE, Bremer

HJ: Letter: Absorption of zinc in acrodermatitis enteropathica. Lancet. 1975;1:85-6.

18. Barnes PM, Moynahan EJ: Zinc deficiency in acrodermatitis enteropathica: multiple dietary intolerance treated with synthetic diet. Proc R Soc Med. 1973;66:327-9.

19. Neldner KH, Hambidge KM: Zinc therapy of acrodermatitis enteropathica. N Engl J Med. 1975;24:879-82.

Copyright by Neerja Puri. This is an open access article distributed under the terms of the Creative Commons Attribution License, which permits unrestricted use, distribution, and reproduction in any medium, provided the original author and source are credited. 